\title{
A Robust Fingerprint Matching Approach: Growing and Fusing of Local Structures
}

\author{
Wenquan Xu, Xiaoguang Chen, and Jufu Feng \\ State Key Laboratory on Machine Perception, \\ Center for Information Science, \\ School of Electronics Engineering and Computer Science, \\ Peking University, Beijing 100871, P.R. China \\ \{xuwq, chenxg,fjf\}@cis.pku.edu.cn
}

\begin{abstract}
This paper proposed a robust fingerprint matching approach based on the growing and fusing of local structures. First, we obtain candidate of minutiae triangles, the smallest local structure in our approach; and then all candidates of minutiae structures grow into larger local structures (we call growing regions) based on which we define the credibility of minutiae triangles and introduce a competition strategy; finally, growing regions compete and fuse into a much larger local structures (called fusion region). The matching score is calculated based on the evaluation of growing and fusing of local structures. Results on FVC2004 show that the proposed approach is robust to fingerprint nonlinear deformation and is efficient.
\end{abstract}

Keywords: fingerprint, nonlinear deformation, robust, local, global, grow, fuse.

\section{Introduction}

The nonlinear deformation of a fingerprint introduced by the elastic feature of the finger and the non-uniform pressure on the finger during the fingerprint acquisition makes finger matching algorithm less efficient and less accurate. Based on the idea that the local may be less affected by the global deformation, a lot of fingerprint matching algorithms adapt the local and global matching scheme. In this scheme we first define the local structure which is at least invariant as to affine transformation; after obtaining candidates of local structures which are possibly matched, we combine them into a global match result. The efficiency of this matching scheme depends on two critical points: first, how reliable the local structure is; second, how well the local match becomes a global match. Many works have been made on the former point. AKJ [2] use a local structure consisting of a minutiae and a list of sampling points on the same ridge of the minutiae. En Zhu[3] constructs a local structure with the orientation information near the minutiae. X. Jiang[4] suggests that the minutiae triangle local structure is less affected by the non-linear deformation. Y.He \&\& J. Tian[5] construct a local structure with two minutiae which he assumes would be more reliable when concerning the information of ridge count. Xuefeng Liang and Tetsuo Asano [8] introduce the minutiae polygons by including more information 
near the bifurcation minutiae. As for the latter point, there is relatively less work. AKJ [2] adapt the explicit alignment method in global matching procedure. Bazen and Gerez [6] introduce an implicit alignment using thin plate template spline. Both explicit and implicit alignments need reference points. Sharat Chikkerur [7] introduces a graph matching algorithm $C B F S$ without alignment. Y. Feng and J. Feng [1] give a definition of local compatibility in an attempt to measure the coherence of local structure pairs and embed it in global matching process.
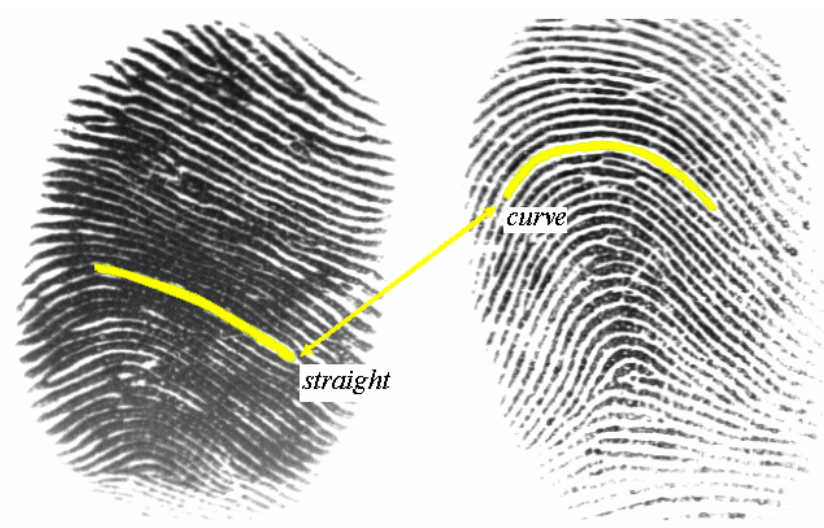

Fig. 1. Two impressions of the same fingerprint (FVC2004 DB1_A 14_3 and 14_6). Because of nonlinear deformation, the ridge in the left impression is closed to a straight line while the correspondent ridge in the right impression is a curve.

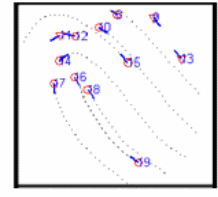

A

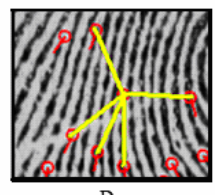

$\mathrm{D}$
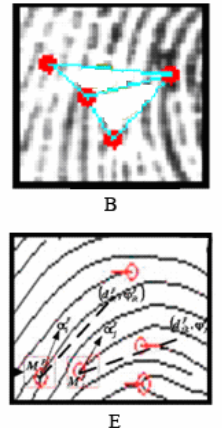

Fig. 2. Minutiae structures: A, minutiae whip; B, minutiae triangle; $\mathrm{C}$, minutiae orientation cross: minutiae structure with local orientation; D, K-plet; E, minutiae stick; F, minutiae polygon.

\section{Proposed Method}

This paper shows the natural way in which local matches grow into global match using a competition strategy. First, candidates of minutiae triangle are obtained; and then we let candidates of minutiae structures grow into larger local structures (called 
growing regions), based on which we define the credibility of each minutiae triangle; finally, growing regions compete and fuse into a much larger local structure (called fusion region). The matching score is calculated based on the evaluation of both the growing region and the fusion region. In this way, we obtain the match result without alignment. The following part of this paper is arranged by the flow of the growing and fusing sequence of local, from minutiae to minutiae triangles, to growing region, to fusion region.

\subsection{Matching of Minutiae Structures}

With minutiae triangle being local structure, we adopt the Delaunay Triangulation method to get minutiae triangles since it is an equiangular triangulation method concerning both of the angle and the length of the triangle, thus making the triangulation more robust for nonlinear deformation. We define a rotation-invariant feature vector:

$$
V_{t r i}=\left(d_{p m}, d_{p n}, \rho_{p}, \rho_{m}, \theta_{p m}, \theta_{p n}\right)
$$

where $d_{p i}$ denotes the distance between minutiae $p$ and minutiae $i, \rho_{p}$ is $\angle m p n$, $\rho_{m}$ is $\angle n m p$ and $\rho_{n}$ is $\angle m n p \quad\left(\rho_{p} \geq \rho_{m} \geq \rho_{n}\right), \theta_{p i}$ denotes the relative radial angle between directions of minutiae $p$ and minutiae $i, \theta_{p m}$ denotes the relative radial angle between directions of minutiae $p$ and minutiae m, as in Fig. 3. Now the distance of minutiae triangles can be defined by the following equation:

$$
D_{t r i}\left(V_{t r i}{ }^{1}, V_{t r i}{ }^{2}\right)=W_{t r i}{ }^{T}\left|V_{t r i}{ }^{1}-V_{t r i}{ }^{2}\right| \text {, }
$$

where $W_{t r i}$ is a $1 \times 6$ weight vector, and $V_{t r i}{ }^{1}$ and $V_{t r i}{ }^{2}$ are feature vectors of two minutiae triangles. We can increase the contribution of the length and the angle of the triangle while at the same time decrease the influence of the orientation of the minutiae by adjusting $W_{t r i}$.

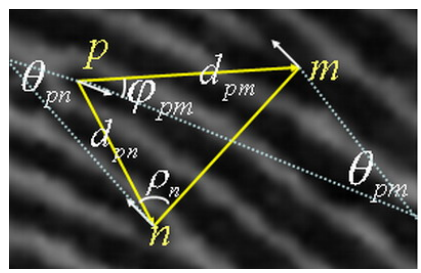

Fig. 3. Minutia triangle constructed by three minutiae $p, m$ and $n$

\subsection{Growing Region and Credibility of Minutiae Structures}

In this stage, the minutiae triangle grows into the growing region, a larger matched local. In according to the similarity of growing regions, we define the credibility of the minutiae structure. 

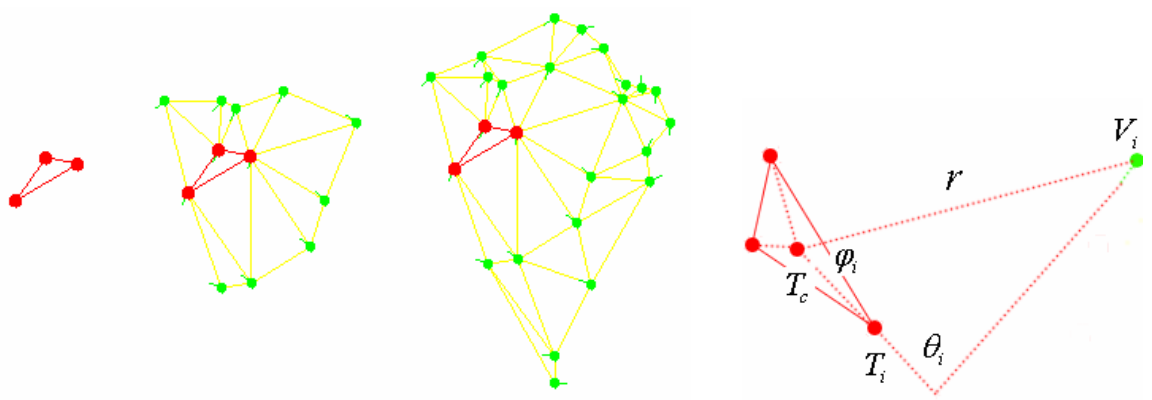

Fig. 4. Left: A core candidate triangle grows into a growing region; Right: The feature vector of a star point in the growing region is defined by the affine transform invariant feature vector $V_{s p}=\left\{\theta_{1}, \theta_{2}, \theta_{3}, \varphi_{1}, \varphi_{2}, \varphi_{3}\right\}$

In the work of Y.S. Feng and J.F. Feng [1], they implicitly use the neighbor minutiae triangle to define the credibility of minutiae triangle. But due to the independent construction of minutiae triangles in template fingerprint and query fingerprint, a candidate triangle can be elected only when their neighbor minutiae triangles are candidate minutiae triangles.

In this paper, we give a more reasonable definition of the credibility of a candidate of minutiae structure with the neighbor minutiae of the minutiae triangle. The growing region is developed from a core candidate triangle by including all the other minutiae triangles that satisfying either one of the following condition: a) the minutiae triangle has at least one common vertex with the core candidate triangle; b) neighboring triangles of the minutiae triangle has at least one common vertex with the core candidate triangle as in Fig. 4 left. For each pair of core candidate triangles, we get a pair of growing regions which is actually a pair of point sets. Calculating the similarity of two growing regions can be treated as calculating the similarity of two point sets. Since the correspondence of the three vertex of the core candidate triangle is known, we can further simplify it to a string matching question. For each Minutiae point ( $V_{i}$, we call it star point) in the growing regions, it can be identified by the affine transformation invariant feature vector:

$$
V_{s p}=\left\{r, \theta_{1}, \theta_{2}, \theta_{3}, \varphi_{1}, \varphi_{2}, \varphi_{3}\right\},
$$

where $r$ is the Euler distance between the star point, $T_{c}$ is the center of the core candidate structure, $\theta_{i}$ is the angle between line $T_{i} T_{c}$ and the minutiae direction of $V_{i}$, and $\varphi_{l}$ is the angle between line $T_{i} T_{c}$ and line $V_{i} T_{c}$ as in Fig.4 right. Then we have the definition of the distance between two star points:

$$
D_{s p}\left(V_{s p}{ }^{1}, V_{s p}{ }^{2}\right)=W_{s p 1}{ }^{T} f\left(V_{s p}{ }^{1}, V_{s p}{ }^{2}\right),
$$




$$
f\left(V_{s p}{ }^{1}, V_{s p}{ }^{2}\right)=\left(\begin{array}{c}
\left|\log r^{1}-\log r^{2}\right| /|\log 0.7| \\
\frac{a b s\left(\theta_{1}{ }^{1}-\theta_{1}{ }^{2}\right)+a b s\left(\theta_{2}{ }^{1}-\theta_{2}{ }^{2}\right)+a b s\left(\theta_{3}{ }^{1}-\theta_{3}{ }^{2}\right)}{3 \times 30} \\
\frac{a b s\left(\varphi_{1}{ }^{1}-\varphi_{1}{ }^{2}\right)+a b s\left(\varphi_{2}{ }^{1}-\varphi_{2}{ }^{2}\right)+a b s\left(\varphi_{3}{ }^{1}-\varphi_{3}{ }^{2}\right)}{3 \times 20}
\end{array}\right),
$$

where $W_{s p 1}$ is a $1 \times 3$ weight vector, and $V_{s p}{ }^{1}$ and $V_{s p}{ }^{2}$ are feature vectors of two star points. We can further define the similarity of two star points as:

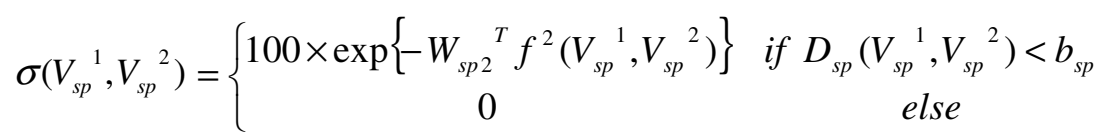

In our experiment on FVC2004, $W_{s p 1}=(1.2,1.2,0.8)^{T}, W_{s p 2}=(0.5,0.5,0.5)^{T}$, and $b_{s p}=1.8$.

We convert the unordered star points of the growing region into an ordered sequence by arranging them in the increasing order of $\varphi_{1}$. And then we adopt the dynamic programming approach [6], which bases on string matching, to get matched number of star points.

The creditability of the core candidate triangle pair is determined by the similarity of growing regions which represent local areas. In our experiment, the creditability of the minutiae structure pair is defined as:

$$
C R=n,
$$

where $n$ is the number of matched minutiae in the pair of growing regions. In this way, the minutiae structure pair gain more credit if it have more matched minutiae in its growing regions. The growing region has a larger area than the minutiae triangle; therefore it is more affected by the nonlinear deformation; but compare to the global region it is still less affected by the nonlinear deformation.

\subsection{Fusing of Growing Regions and the Compatibility of Minutiae Structure}

Human experts usually pay special attention to the interrelation of some candidate minutiae structures and double check the neighborhood of the minutiae structure. In order to imitate this human behavior, we propose a measurement for the coherence of local structure pairs, called local structure compatibility. In according to the compatibility of minutiae structures, all growing regions are fused into a fusion region. The fusing process is done by a majority voting among candidate local structures during which candidate local structures compete against each other. 


\subsubsection{The Compatibility of Minutiae Structure}

Some candidates of minutiae triangle pairs we get from the local matching process may not be compatible. They are not compatible because of the relative position or relative pose or both. In order to depict this difference, we first define the feature vector of two minutiae structure as:

$$
V_{m m}=\left\{r, \varphi_{1}, \varphi_{2}, \varphi_{3}, \theta_{1}, \theta_{2}, \theta_{3}\right\}
$$

where $r$ is the distance between minutiae triangles, $\varphi_{i}$ is the angle from line $p_{c} m_{c}$ to the line $p_{c} p_{i}, \theta_{i}$ is the angle from line $p_{c} m_{c}$ to line $m_{c} m_{i}$ as in Fig. 5 left. Then we define the compatibility of minutiae triangles as:

$$
C O=G\left(W \cdot\left|V_{m m}^{1}-V_{m m}^{2}\right|\right),
$$

where $G(x)$ is monotonically decreasing function and $W$ is a weight vector. We simply choose $G(x)$ as:

$$
G(x)=\left\{\begin{array}{ll}
1 & x<b_{c o} \\
0 & \text { else }
\end{array} .\right.
$$

where $b_{c o}$ is predefined boundary. Since $V_{m m}$ only accounts for the Euler distance of two minutiae triangles, it can not discriminate the topological difference of two pairs of minutiae triangles. Therefore, we define three types of topological condition to check the compatibility of minutiae triangles as in Fig. 5 right: A, separate; B, share one vertex; $\mathrm{C}$, share two vertices.
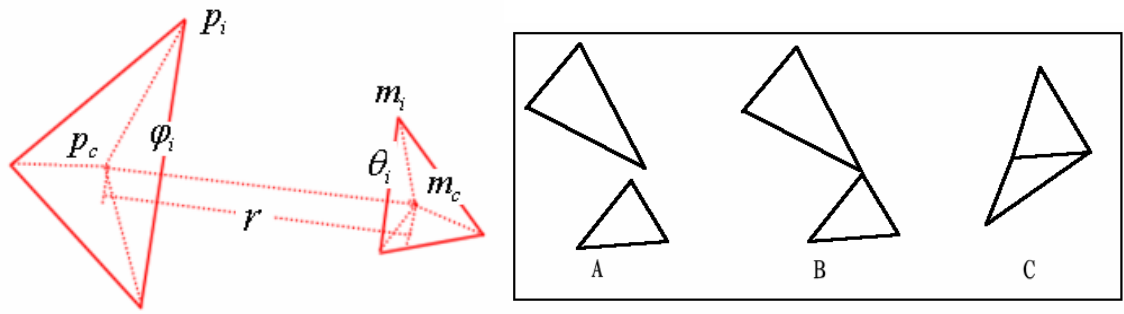

Fig. 5. Left, the feature vector of two triangle local structure can be defined by $\left\{r,\left\{\varphi_{i}\right\},\left\{\theta_{i}\right\}\right\}$; Right, three kinds of topological conditions to check the compatibility of minutiae triangles: A, separate; $\mathrm{B}$, share one vertex; $\mathrm{C}$, share two vertices.

\subsubsection{Majority Voting Among Minutiae Structure}

In the fusing process, the possibly largest group of minutiae structures that are most compatible with each other are selected. We hold the majority voting with competition strategy, in which every pair of minutiae structures scores another one and only those candidate minutiae structure pairs that have a score larger than a certain value $b_{m v}$ can survive and be fused into a global match region. And then all 
three vertices of compatible minutiae triangles together with their matched neighbor minutiae are labeled as matched minutiae. Sometimes a minutia in the template fingerprint may several possible matched minutiae in the query fingerprint obtained by different LMR. In this case, these minutiae correspondences are not reliable and are omitted.

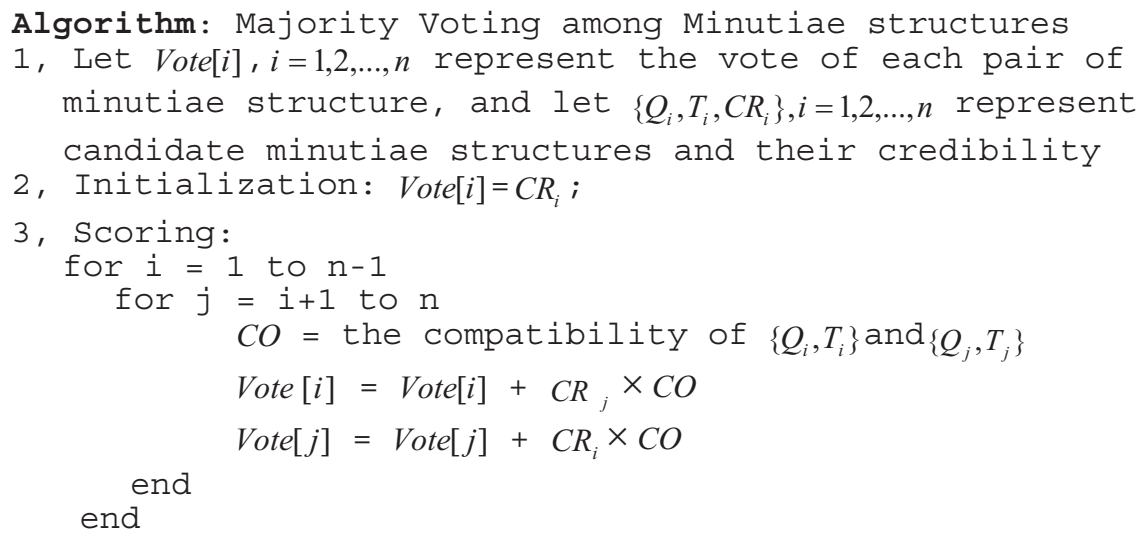

After the fusing process, all the compatible growing regions are fused into a larger local (called fusion region). The fusion region is a circle area that can just hold all the matched minutiae inside.

\subsubsection{Evaluation of the Fusion Region}

The fusion region is actually a local area but larger than the growing region. The evaluation here uses the information of unmatched minutiae in the fusion region which minimize the false matched rate. It is based on the following situations we observed: 1, all growing regions in a genuine match tend to be fused into a connected matched region. However in an imposter match, growing regions may be fused into several separate matched regions. That is, almost all minutiae located in the fusion region are matched minutiae in a genuine match. While in imposter matches, there are a good portion of unmatched minutiae are located in the fusion region; 2, besides, the deformation of the fusion region is consistent in a genuine match, either squeezing, tensing, or rotating in the same direction. And it is not true for an imposter match.

A critical function is used to evaluate the fusing result:

$$
C_{G M A}=\frac{n}{\max \left(n_{f t}, n_{f q}\right)},
$$

where $\mathrm{n}$ is the number of matched minutiae between the template fingerprint and the query fingerprint, and $n_{f t}$ and $n_{f q}$ are the numbers of minutiae located in the fusion region in the template fingerprint and query fingerprint correspondingly. 


\subsection{Scoring}

The scoring process uses the information lying in the local as well as in the global to measure the similarity of local structures when they grow. The match score is calculated as:

$$
\text { Score }=100 \frac{n^{2}}{n_{t} n_{q}} n_{L M R} C_{G M R},
$$

where $n_{L M A}$ is the number of growing regions and $C_{G M R}$ is the evaluation of the fusion region.

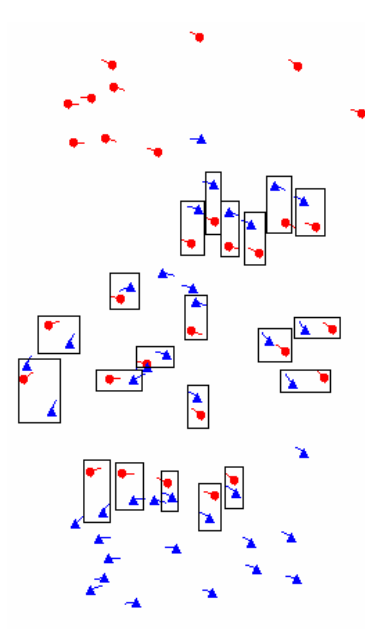

A

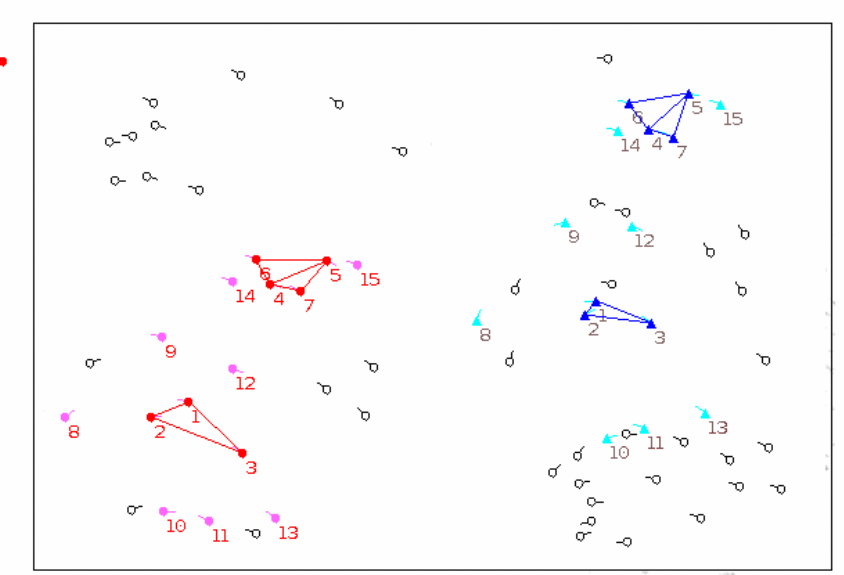

B
$\mathrm{C}$

Fig. 6. Comparing the matching result of fingerprints with large nonlinear deformation between the alignment base method and our method: A, shows minutiae correspondence of two impressions of a fingerprint after alignment manually; $\mathrm{B}$ and $\mathrm{C}$, show minutiae correspondences obtained by our approach

Table 1. Result of our method on the database of FVC2004

\begin{tabular}{lllll}
\hline $\begin{array}{l}\text { Database } \\
\text { FVC2004 }\end{array}$ & EER $(\%)$ & $\begin{array}{l}\text { FMR100 } \\
(\%)\end{array}$ & $\begin{array}{l}\text { FMR1000 } \\
(\%)\end{array}$ & $\begin{array}{l}\text { ZeroFMR } \\
(\%)\end{array}$ \\
\hline DB1_a & 3.819 & 7.363 & 7.354 & 12.200 \\
DB2_a & 4.712 & 8.854 & 8.961 & 15.769 \\
DB3_a & 2.923 & 4.201 & 4.249 & 7.592 \\
\hline
\end{tabular}

\section{Experiments and Results}

Our experiment is performed on fingerprint databases of FVC2004 (DB1_A, DB2_A, DB3_A). Each of the three fingerprint databases has 8 fingerprints of 100 fingers 
totally 800 fingerprints. This dataset is available on website of FVC 2004, and is proved to be the most difficult for most methods because no efforts were made to control image quality and the sensor platens were not systematically cleaned [9]. And we take the standard performance evaluation indicators of FVC: genuine and imposter score distribution, equal error rate (EER) and ROC [10].

With only minutiae information, our approach has EER of 3.8\%, 4.7\%, 2.9\% on the three databases of FVC2004 (DB1_a, DB2_a, DB3_a correspondingly). The average processing time is 0.03 seconds per matching on an AMD64 3200+ 1GB PC under Windows 2003. There are two impression of a fingerprint from FVC2004 DB1_A (14_3 14_6) as in Fig.1. Because of nonlinear deformation, the ridge in the left impression is approximately a straight line while the correspondent ridge in the right impression is curve. The minutiae correspondences are pointed out manually after alignment in Fig.6 left from which we can see that it is hard to find the correspondence using an alignment based fingerprint matching algorithm. But with our approach, we can find almost all the minutiae correspondence located in the overlap region.

\section{Conclusion and Future Work}

In the paper, we present a robust fingerprint matching approach in which fingerprints are matched by local in three stages. Locals grow and compete in each stage and gradually become a global region. As a result, there is no alignment. Results of our experiment on FVC2004 shows that our approach is robust to the nonlinear deformation and has a low false accept rate.

The triangulation process is the first step of our approach and is thus the most important stage. Actually it is a state-of-art problem for several reasons: $C_{n}^{3}$ different triangles can be generated from $n$ minutiae; triangle set of too large size has a high computational and memory complexity; triangle set of too small size often fails to have common triangles. In our experiment the Delaunay Triangulation can get a more reliable triangle set than the k-neighbor method [1][4].

The evaluation and the growing strategy applied to the matched local estimate how well locals match. So it has to account for the deformation of fingerprint which unfortunately lacks efficient description. In our method we skip this problem by stress more on the number of matched minutiae. In future work, we will understand the nonlinear deformation of fingerprint and give a more reasonable definition of the matched local.

Acknowledgments. This work was supported by NSFC(60575002、60635030) and NKBRPC (2004CB318000) and Program for New Century Excellent Talents in University. Thanks all reviewers for their useful comments.

\section{References}

1. Feng, Y., Feng, J.: A Novel Fingerprint Matching Scheme Based on Local Structure Compatibility. In: ICPR2006, vol. 4, track 4, Thu-O-II-1b (2006)

2. Jain, A., Hong, L.: On-line fingerprint verification. IEEE Trans. PAMI. 19(4), 302-314 (1997) 
3. Zhu, E.: Fingerprint matching based on global alignment of multiple reference minutiae. Pattern Recognition 38, 1685-1694 (2005)

4. Jiang, X., Yau, W.-Y.: Fingerprint minutiae matching based on the local and global structures. In: ICPR2000, vol. 2, pp. 1038-1041 (2000)

5. He, Y., Tian, J.: Fingerprint Matching Based on Global Comprehensive Similarity. IEEE Trans. PAMI 28(6), 850-862 (2006)

6. Bazen, A.M., Gerez, S.H.: Fingerprint matching by thin-plate spline modeling of elastic deformations. Pattern Recognition 36, 1859-1867 (2003)

7. Chikkerur, S., Govindaraju, V., Cartwright, A.N.: K-plet and Coupled BFS: A Graph Based Fingerprint Representation and Matching Algorithm. In: ICB2006 (2006)

8. Liang, X., Asano, T.: Fingerprint Matching Using Minutia Polygons. In: ICPR2006, vol. 1, track 4, Mon-O-IV-2 (2006)

9. Fingerprint Verification Competition: (FVC2004) (2004), http://bias.csr.unibo.it/fvc2004

10. Cappelli, R., Maio, D., Maltoni, D., Wayman, J., Jain, A.K.: Performance Evaluation of Fingerprint verification systems. IEEE Trans. PAMI. 28(1), 3-18 (2006) 